\title{
Hypergraph coverings and their zeta functions*
}

\author{
Deqiong Li \\ College of Mathematics and Statistics \\ Hunan Normal University \\ Changsha, Hunan 410081, China \\ Department of Mathematics \\ Hunan University of Commerce \\ Changsha, Hunan 410205, China \\ deqiongli@126.com
}

\author{
Yaoping $\mathrm{Hou}^{\dagger}$ \\ Key Laboratory of HPCSIP \\ College of Mathematics and Statistics \\ Hunan Normal University \\ Changsha, Hunan 410081, China \\ yphou@hunnu.edu.cn
}

Submitted: Mar 31, 2018; Accepted: Dec 5, 2018; Published: Dec 21, 2018

(C) The authors. Released under the CC BY-ND license (International 4.0).

\begin{abstract}
Let $H$ be a finite hypergraph. The concept of hypergraph coverings over $H$ is introduced, and we can generate all hypergraph coverings by permutation voltage assignments of the edge-colored graph or the incidence graph of $H$. Consequently, we show two explicit decomposition formulae for the zeta function of any hypergraph covering $\bar{H}$ over $H$ which indicates that the zeta function of $H$ divides the zeta function of $\bar{H}$.
\end{abstract}

Mathematics Subject Classifications: 05C50,05C65, 05C25

\section{Introduction}

Let $G=\left(V_{G}, E_{G}\right)$ be a finite graph which multiple edges and loops are allowed. $\overleftrightarrow{G}$ is the symmetric digraph corresponding to $G$ which is obtained from $G$ by changing every undirected edge of $G$ to a pair of oppositely directed edges (i.e., arcs). We denote the arc from the vertex $u$ to the vertex $v$ as ordered pair $(u, v)$. For $e=(u, v) \in E_{\overleftrightarrow{G}}$, set $u=o(e)$ and $v=t(e)$, and $e^{-1}=(v, u)$ means the reverse arc to $e$.

A covering projection from a graph $\bar{G}$ to $G$ is a surjection $\pi: V_{\bar{G}} \rightarrow V_{G}$ such that $\left.\pi\right|_{N(\bar{v})}: N(\bar{v}) \rightarrow N(v)$ is a bijection for all vertices $v \in V_{G}$ and $\bar{v} \in \pi^{-1}(v)$, where $N(v)$, the neighborhood of the vertex $v$, is the set of vertices adjacent to $v$. The graph $\bar{G}$ is called a graph covering over $G$, and $\bar{G}$ is a $k$-fold covering if $\pi$ is $k$-to-one. As an important class of graphs, covering graphs have been studied in many literatures, such as $[12,13,15]$.

*Supported by the National Natural Science Foundation of China (No. 11571101).

${ }^{\dagger}$ Corresponding author 
Let $S_{k}$ be the symmetric group on $[k]=\{1,2, \ldots, k\}$. The function $\phi: E_{\overleftrightarrow{G}} \rightarrow S_{k}$ is called a permutation voltage assignment on $G$ if $\phi(e)^{-1}=\phi\left(e^{-1}\right)$ for each $e \in E \overleftrightarrow{G}$. The permutation voltage assignment $\phi$ is trivial if $\phi(e)^{-1}=\phi\left(e^{-1}\right)=1$ for each $e \in E \overleftrightarrow{G}$, where 1 is the identity of $S_{k}$. Otherwise, $\phi$ is nontrivial. The pair $(G, \phi)$ is called a permutation voltage graph. A derived graph $G^{\phi}$ of $(G, \phi)$ is a graph with the vertex set $V_{G^{\phi}}=V_{G} \times[k]=\left\{(v, i) \mid v \in V_{G}, i \in[k]\right\}$, and $(v, i)$ and $(u, j)$ are adjacent if and only if $e=(v, u) \in E_{\overleftrightarrow{G}}$ and $i=\phi(e) j$. Gross and Tucker found the following lemma:

Lemma 1. [2] There is a one-to-one correspondence between each $k$-fold covering $\bar{G}$ over $G$ and some derived graph $G^{\phi}$ of $(G, \phi)$.

The zeta function $[3,12]$ of a finite graph $G$ is defined to be the function of a complex number $u$ with $|u|$ sufficiently small, given by

$$
\varsigma_{G}(u)=\prod_{[C]}\left(1-u^{|C|}\right)^{-1}
$$

where $[C]$ runs over all equivalence classes of prime, reduced cycles of $G$. We refer the reader to [16] for an in-depth treatment of the zeta functions of graphs.

The degree of the vertex $v_{i}$ in the graph $G$ is denoted by $d_{G}\left(v_{i}\right)$. Let $A(G)$ and $D(G)$ denote the adjacency matrix and the degree-diagonal matrix of the graph $G$, respectively. Bass [1] proved that the zeta function of a graph $G$ with minimum degree 2 satisfies the following determinant formula

$$
\varsigma_{G}(u)^{-1}=\left(1-u^{2}\right)^{\left|E_{G}\right|-\left|V_{G}\right|} \operatorname{det}\left(I_{n}-u A(G)+u^{2} Q(G)\right),
$$

where $I_{n}$ is the identity matrix with order $n$ and $Q(G)=D(G)-I_{n}$.

By Eq. (1), Terras [16] showed the zeta function of a finite graph divides the zeta function of any covering over this graph. Moreover, Mizuno and Sato in [7] gave an explicit composition formula for the zeta function of any covering over a finite graph by applying Eq. (1) and the representation theory of symmetric groups. More results on the zeta functions on graph coverings can see $[5,7,8]$.

Subsequently, Storm [14] introduced the definition of the zeta function of a hypergraph being thought of the generalization of the zeta function of a graph. Let $H=\left(V_{H}, E_{H}\right)$ be a hypergraph on the vertex set $V_{H}$, and $E_{H}$ is a set of non-empty subsets of $V_{H}$ called hyperedges. A hyperpath $P$ in $H$ is a sequence $P=\left(x=v_{1}, e_{1}, v_{2}, e_{2}, v_{3}, \ldots, e_{t}, v_{t+1}=y\right)$ of $t+1$ vertices and $t$ hyperedges such that $v_{j} \in V_{H}, e_{j} \in E_{H}, v_{1} \in e_{1}, v_{t+1} \in e_{t}$ and $v_{i} \in e_{i}, e_{i-1}$ for each $i=2, \ldots, t$. The length of $P$ is $|P|=t$. The hyperpath $P$ has a hyperedge backtrack if there is a subsequence of $P$ of the form $(e, v, e)$. If $x=v_{1}=v_{t+1}=y$ the hyperpath $P$ is called a closed hyperpath or hypercycle. Let $C^{m}$ denote the hypercycle obtained by going $m$ times around the hypercycle $C$. A closed hyperpath $C$ is tail-less if $C^{2}$ does not have a hyperedge backtrack, and a closed hyperpath $P$ is reduced if it has no hyperedge backtrack or tail. A hypercycle $C$ is said to be prime if $C \neq B^{m}$ for any hypercycle $B$ and integer $m \geqslant 2$. Finally, two hypercycles $C_{1}=\left(v_{1}, e_{1}, v_{2}, e_{2}, v_{3}, \ldots, e_{t}, v_{1}\right)$ and $C_{2}=\left(w_{1}, f_{1}, w_{2}, f_{2}, w_{3}, \ldots, f_{t}, w_{1}\right)$ are called equivalent if $w_{j}=v_{j+k}$ and $f_{j}=e_{j+k}$ for 
all $j$, i.e., $C_{1}$ is a cyclic permutation of $C_{2}$. The equivalence class containing $C$ is denoted by $[C]$.

For $u \in \mathbb{C}$ with $|u|$ sufficiently small, the (generalized Ihara-Selberg) zeta function of a finite hypergraph $H$ is defined by

$$
\varsigma_{H}(u)=\prod_{[C]}\left(1-u^{|C|}\right)^{-1},
$$

where $[C]$ runs over all equivalence classes of prime and reduced hypercycles of $H$. The zeta function of a hypergraph in the above form is generally an infinite product.

There are several methods in which a hypergraph can be transformed into a graph representation. For example, a hypergraph can be transformed into the edge-colored graph, the oriented line graph and the incidence graph of it. The incidence graph $B_{H}$ of $H$ is a bipartite graph with the vertex set $V_{B_{H}}=V_{H} \cup E_{H}$, and $v \in V_{H}$ and $e \in E_{H}$ are adjacent if and only if $v \in e$. Besides, we will construct two edges between $v$ and $e$ in $B_{H}$ when $e$ is a loop at the vertex $v$ in $H$. The hyperedge set of a hypergraph $H$ is labelled as $E_{H}=\left\{e_{1}, \ldots, e_{m}\right\}$ and fix $m$ colors $\left\{c_{1}, \ldots, c_{m}\right\}$. The edge-colored graph $G H_{c}$ of $H$ is constructed as follows: The vertex set is the vertex set of $H$, and for each hyperedge $e_{i} \in E_{H}$, a $\left|e_{i}\right|$-clique (i.e., the complete graph with $\left|e_{i}\right|$ vertices) on the vertices in $e_{i}$ is constructed such that all edges of the $\left|e_{i}\right|$-clique are colored by $c_{i}$. In addition, a loop is added at the vertex $v$ in $G H_{c}$ if the hyperedge of $H$ is a loop at $v$. Here, it should be noted that the definitions of the incidence graph and the edge-colored graph of a hypergraph are a little bit different from those in [14] where loops in a hypergraph are not taken into account. For example, the hypergraph $H$ and its edge-colored graph $G H_{c}$ is showed in Figure 1. Let $\overleftrightarrow{G H_{c}}$ be the symmetric digraph corresponding to $G H_{c}$ and

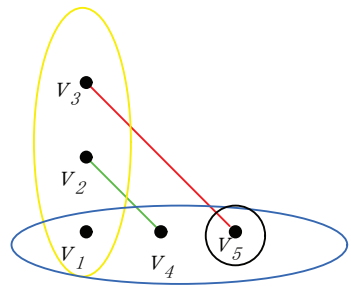

$H$

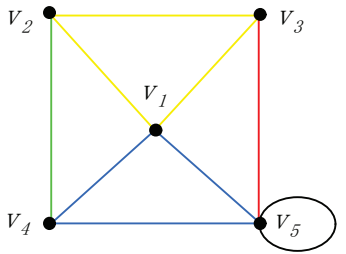

$\mathrm{GH}_{c}$

Figure 1: The hypergraph $H$, the edge-colored graph $G H_{c}$ of $H$

color the arcs in $\overleftrightarrow{G H_{c}}$ keeping consistent to undirected edge of $G H_{c}$ they are induced from. Let $H_{L}$ be the oriented line graph of $H$ defined by $V_{H_{L}}=E_{\overleftrightarrow{G H_{c}}}$ and $E_{H_{L}}=\left\{\left(e_{i}, e_{j}\right) \in\right.$ $\left.E_{\overleftrightarrow{G H_{c}}} \times E_{\overleftrightarrow{G H_{c}}}: c\left(e_{i}\right) \neq c\left(e_{j}\right), t\left(e_{i}\right)=o\left(e_{j}\right)\right\}$, where $c\left(e_{i}\right)$ is the colored assigned to the arc $e_{i} \in E_{\overleftrightarrow{G H_{c}}} . A\left(H_{L}\right)=\left(a_{i j}\right)$ is the adjacency matrix of $H_{L}$, i.e., $a_{i j}=1$ if there is an arc from the vertex $e_{i}$ to the vertex $e_{j}$, and $a_{i j}=0$ otherwise. In terms of the oriented line graph and the incidence graph of a hypergraph, Storm [14] gave two explicit determinant expressions for the Ihara-Selberg zeta function of a hypergraph utilizing the results of Kotani and Sunada [3] and Bass [1]. 
Theorem 2. [14] Let $H$ be a finite connected hypergraph without loops, and each vertex of $H$ is in at least two hyperedges. Then we have

$$
\begin{aligned}
& \varsigma_{H}(u)^{-1} \\
= & \operatorname{det}\left(I-u A\left(H_{L}\right)\right) \\
= & \varsigma_{B_{H}}(\sqrt{u})^{-1}=(1-u)^{\left|E_{B_{H}}\right|-\left|V_{B_{H}}\right|} \operatorname{det}\left(I_{\left|V_{B_{H}}\right|+\left|E_{B_{H}}\right|}-\sqrt{u} A\left(B_{H}\right)+u Q\left(B_{H}\right)\right) .
\end{aligned}
$$

Another determinant expression of the zeta function of a hypergraph is characterized in [9]. In addition, Sato [10] introduced the edge zeta function of a hypergraph and presented its determinant expression, and a decomposition formula for the edge zeta function of a group covering of a hypergraph was given.

In view of the above, in Section 2 we generalize the definition of graph coverings over a graph to hypergraphs. By permutation voltage assignments of the edge-colored graph or the incidence graph of a finite hypergraph, we can generate all hypergraph coverings. In Section 3, based on the representation theory of symmetric groups we find two explicit decomposition formulae for the zeta function of any hypergraph covering which indicates the zeta function of any hypergraph covering can be expressed by the zeta function of this hypergraph.

\section{Hypergraph coverings over a finite hypergraph}

Let $H=\left(V_{H}, E_{H}\right)$ be a finite hypergraph. We allow hyperedges to repeat and allow loops. $E_{v}$ is the hyperedge set containing the vertex $v$. The neighborhood $N(v)$ of a vertex $v$ in $H$ is the set of vertices $u$ such that $u$ and $v$ are adjacent, i.e., $N(v)=\left\{u \in V_{H}: u \in\right.$ $e$ and $\left.e \in E_{v}\right\}$.

A homomorphism from a finite hypergraph $\bar{H}$ to $H$ is a map which sends vertices to vertices, hyperedges to hyperedges, and preserves the adjacency. A homomorphism map $\pi: \bar{H} \rightarrow H$ is a hypergraph covering projection if it is surjection such that $\left.\pi\right|_{N(v)}: N(\widetilde{v}) \rightarrow$ $N(v)$ is a bijection for all vertices $v \in V_{H}$ and $\widetilde{v} \in \pi^{-1}(v)$. The hypergraph $\bar{H}$ is called a hypergraph covering over $H$, or a covering for short. If $\pi$ is $k$-to-one, we call $\bar{H}$ a $k$-fold hypergraph covering. It should be pointed out that in the special case where each of hyperedges contains only one vertex or two vertices, a hypergraph covering reduces to a graph covering. From the definition of $k$-fold hypergraph coverings, each vertex $v$ and each hyperedge $e$ of $H$ have $k$ vertices and $k$ hyperedges in their respectively preimages $\pi^{-1}(v)$ and $\pi^{-1}(e)$. Also, the hypergraph covering over $H$ is not unique. As an illustration, we give two 2-fold hypergraph coverings over the hypergraph $H$. See Figure 2.

Let $\bar{H}$ be a $k$-fold hypergraph covering over $H$. Let $G \bar{H}_{c}$ and $B_{\bar{H}}$ be the edge-colored graph and the incidence graph of $\bar{H}$, respectively. By applying the definition of hypergraph coverings, it is easy to find that $G \bar{H}_{c}$ and $B_{\bar{H}}$ must be the $k$-fold graph coverings over $G H_{c}$ and $B_{H}$, respectively.

Now, we shall generate all hypergraph coverings using two distinct methods.

Firstly, we establish a one-to-one correspondence on any $k$-fold hypergraph covering over the hypergraph $H$ with the help of the edge-colored graph of $H$. The hyperedge 


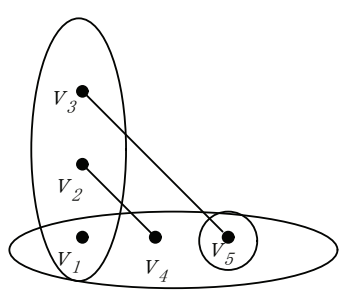

$H$
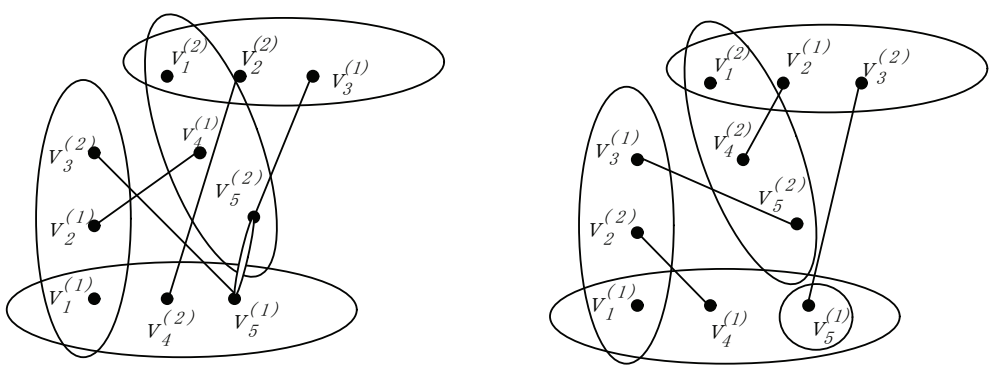

Figure 2: The hypergraph $H$ and two 2-fold hypergraph coverings over $H$

set of $H$ is labelled as $E_{H}=\left\{e_{1}, \ldots, e_{m}\right\}$ and fix $m$ colors $\left\{c_{1}, \ldots, c_{m}\right\}$. An additional condition on $k$-fold coverings over $H$ is assumed: For any hypergraph covering projection from the hypergraph $\bar{H}$ to $H$, any hyperedge of $\bar{H}$ has the same color of its image. With the same method, we color all edges of any $k$-fold covering over $G H_{c}$.

Lemma 3. Let $K_{n}$ be a complete graph with $n(n \geqslant 3)$ vertices. Then there exists a nontrivial permutation voltage assignment $\phi: E_{\widehat{K}_{n}} \rightarrow S_{k}(k>1)$ on $K_{n}$ such that $\phi\left(\left(v_{i}, v_{j}\right)\right)$ $\phi\left(\left(v_{j}, v_{t}\right)\right) \phi\left(\left(v_{t}, v_{i}\right)\right)=1$ for every three distinct vertices $v_{i}, v_{j}, v_{t}$ of $K_{n}$.

Proof. Let $T_{n}$ be a spanning star of $K_{n}$ with $n-1$ edges $v_{1} v_{2}, v_{1} v_{3}, \ldots, v_{1} v_{n}$. Furthermore, we give a permutation voltage assignment $\phi\left(\left(v_{1}, v_{i}\right)\right)$ on the arc $\left(v_{1}, v_{i}\right)$ arbitrarily for $i=2, \ldots, n$. Then we decide $(n-1)(n-2) / 2$ values $\phi\left(\left(v_{i}, v_{j}\right)\right)$ on the arc $\left(v_{i}, v_{j}\right)(2 \leqslant$ $i<j \leqslant n)$ as follows:

$$
\phi\left(\left(v_{i}, v_{j}\right)\right)=\phi\left(\left(v_{i}, v_{1}\right)\right) \phi\left(\left(v_{1}, v_{j}\right)\right) .
$$

Note that $\phi\left(e^{-1}\right)=\phi^{-1}(e)$ for any arc $e \in E_{\overleftrightarrow{K_{n}}}$. Thus, for every triangle with vertices $v_{i}, v_{j}$ and $v_{t}(2 \leqslant i<j<t \leqslant n)$ of $K_{n}$ we have

$$
\begin{aligned}
& \phi\left(\left(v_{i}, v_{j}\right)\right) \phi\left(\left(v_{j}, v_{t}\right)\right) \phi\left(\left(v_{t}, v_{i}\right)\right) \\
= & \phi\left(\left(v_{i}, v_{1}\right)\right) \phi\left(\left(v_{1}, v_{j}\right)\right) \phi\left(\left(v_{j}, v_{1}\right)\right) \phi\left(\left(v_{1}, v_{t}\right)\right) \phi\left(\left(v_{t}, v_{1}\right)\right) \phi\left(\left(v_{1}, v_{i}\right)\right) \\
= & 1 .
\end{aligned}
$$

The proof is completed.

The assignment $\phi: E_{\overleftrightarrow{G H_{c}}} \rightarrow S_{k}$ of permutation voltage on $G H_{c}$ such that $\phi\left(\left(v_{i}, v_{j}\right)\right)$ $\phi\left(\left(v_{j}, v_{t}\right)\right) \phi\left(\left(v_{t}, v_{i}\right)\right)=1$ for any three distinct arcs with the same color, denoted by $\widetilde{\phi}$, is called the strong permutation voltage assignment on $G H_{c}$. Obviously, $\widetilde{\phi}$ is feasible from Lemma 3. Notice that $\widetilde{\phi}$ is a general permutation voltage assignment $\phi$ if edges in $G H_{c}$ with the same color are only an edge or a loop. Let $C(\phi ; k)$ denote the set of all strong permutation voltage assignments $\widetilde{\phi}: E_{\overleftarrow{G H_{c}}} \rightarrow S_{k}$. From the construction of $\widetilde{\phi}$ and the definition of the covering projection, it is easy to check that the preimage of any clique (in $G H_{c}$ ) with identical color is $k$ cliques. Moreover, we assign the same color to these $k$ 

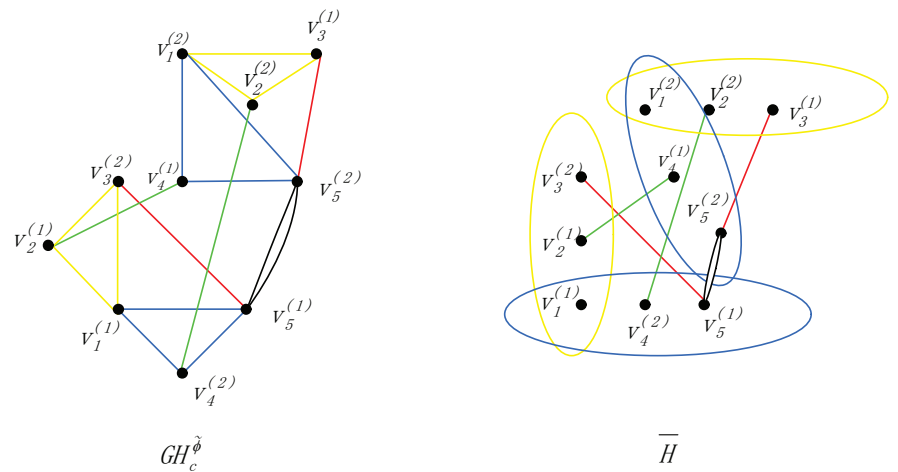

Figure 3: The derived graph $G H_{c}^{\widetilde{\phi}}$, and the corresponding 2-fold hypergraph coverings $\bar{H}$ over $H$

cliques as their image. Subsequently, the hypergraph $H^{\widetilde{\phi}}$ associated with $\widetilde{\phi}$ is established as follows: The vertex set $H^{\widetilde{\phi}}$ is the vertex set of the derived graph $G H_{c}^{\widetilde{\phi}}$ of $\left(G H_{c}, \widetilde{\phi}\right)$. For each clique with identical color in $G H_{c}^{\widetilde{\phi}}$ a hyperedge is formed on the vertices of this clique, and a loop is formed on the vertex where there exists a loop in $G H_{c}^{\widetilde{\phi}}$. $H^{\widetilde{\phi}}$ is called the derived hypergraph associated with the strong permutation voltage assignment $\widetilde{\phi}$. From the construction of $H^{\widetilde{\phi}}$, it is apparent that $H^{\widetilde{\phi}}$ must be a $k$-fold hypergraph covering over $H$ and $G H_{c}^{\widetilde{\phi}}$ can be considered as the edge-colored graph of $H^{\widetilde{\phi}}$.

Theorem 4. Let $\bar{H}$ be any $k$-fold hypergraph covering over the hypergraph $H$. Then we can establish a one-to-one correspondence between $\bar{H}$ and some derived hypergraph $H^{\widetilde{\phi}}$ associate with $\widetilde{\phi} \in C(\widetilde{\phi} ; k)$.

Proof. Since the edge-colored graph $G \bar{H}_{c}$ of $\bar{H}$ is a $k$-fold covering over $G H_{c}$, then by Lemmas 1 and 3, there exists a strong permutation voltage assignment $\widetilde{\phi}$ on $G H_{c}$ such that the derived graph $G H_{c}^{\widetilde{\phi}}$ associated with $\widetilde{\phi}$ is corresponding to $G \bar{H}_{c}$. The proof is completed by noting that every hypergraph is one-to-one corresponding to its edge-colored graph.

Example 5. In Figure 1, we assign the strong permutation voltage $\widetilde{\widetilde{\phi}} \underset{\widetilde{\phi}}{\text { on }} G H_{c}$ as follows: $\widetilde{\phi}\left(\left(v_{2}, v_{3}\right)\right)=\widetilde{\phi}\left(\left(v_{4}, v_{5}\right)\right)=\widetilde{\phi}\left(\left(v_{1}, v_{3}\right)\right)=\widetilde{\phi}\left(\left(v_{3}, v_{5}\right)\right)=\widetilde{\phi}\left(\left(v_{1}, v_{4}\right)\right)=\widetilde{\phi}\left(\left(v_{5}, v_{5}\right)\right)=(12)$, $\widetilde{\phi}\left(\left(v_{1}, v_{2}\right)\right)=\widetilde{\phi}\left(\left(v_{1}, v_{5}\right)\right)=\widetilde{\phi}\left(\left(v_{2}, v_{4}\right)\right)=1$. Then the derived graph $G H_{c}^{\widetilde{\phi}}$ and the corresponding 2-fold hypergraph covering $\bar{H}$ over $H$ are obtained, see Figure 3.

Next, we find another method to establish a representation to all $k$-fold hypergraph coverings over a finite hypergraph. For a finite hypergraph $H$ with the vertex set $V_{H}=$ $\left\{v_{1}, v_{2}, \ldots, v_{n}\right\}$ and the hyperedge set $E_{H}=\left\{e_{1}, e_{2}, \ldots, e_{m}\right\}$. Suppose that $\phi: E_{\overleftrightarrow{B_{H}}} \rightarrow S_{k}$ is a permutation voltage assignment on the incidence graph $B_{H}$ of $H$. It is known that the derived graph $B_{H}^{\phi}$ of $\left(B_{H}, \phi\right)$ brings into a correspondence with some $k$-fold covering over $B_{H}$. The derived hypergraph $H^{B_{H}^{\phi}}$ associated with $B_{H}^{\phi}$ is constructed as follows: The vertex set $V_{H_{H}^{B_{H}^{\phi}}}=\left\{\left(v_{i}, t\right) \mid v_{i} \in V_{H}, t \in[k]\right\}$, and the vertices $\left(v_{i}, t\right)$ and $\left(v_{j}, s\right)$ are adjacent 
if and only if there is a vertex $\left(e_{l}, h\right)$ in $B_{H}^{\phi}$ such that $\phi\left(\left(v_{i}, e_{l}\right)\right) t=h$ and $\phi\left(\left(e_{l}, v_{j}\right)\right) h=s$. That is to say all vertices are adjacent to $\left(e_{l}, h\right)$ in $B_{H}^{\phi}$ form a hyperedge of $H^{B_{H}^{\phi}}$. It is worth nothing that a loop is formed at the vertex $\left(v_{i}, t\right)$ in $H^{B_{H}^{\phi}}$ if $\left(v_{i}, t\right)=\left(v_{j}, s\right)$. Obviously, $H^{B_{H}^{\phi}}$ is a $k$-fold hypergraph covering over $H$ by the construction of $H^{B_{H}^{\phi}}$. Thus, for each permutation voltage assignment $\phi$ on $B_{H}$, there must exist a $k$-fold hypergraph covering $\bar{H}$ over $H$ corresponding to $H^{B_{H}^{\phi}}$. On the other hand, the graph $B_{H}^{\phi}$ is considered as the incidence graph of $H^{B_{H}^{\phi}}$ if the hyperedge containing the vertices $\left(v_{i}, t\right)$ and $\left(v_{j}, s\right)$ is labelled to $\left(e_{l}, h\right)$. However, different permutation voltage assignments on $B_{H}$ may lead to the same derived hypergraph $H_{H}^{B_{H}^{\phi}}$ because the vertex $\left(e_{l}, h\right)$ in $B_{H}^{\phi}$ transforms into a hyperedge when $B_{H}^{\phi}$ acts as a graph representation of $H^{B_{H}^{\phi}}$. Therefore, each hypergraph covering over $H$ can be generated by at least one permutation voltage assignment $\phi$ on $B_{H}$.

Example 6. In Figure 4, $B_{H}$ is the incidence graph of the hypergraph $H$. The permutation voltage assignments $\phi_{1}$ and $\phi_{2}$ on $B_{H}$ can generate two distinct derived graphs $B_{\underline{H}}^{\phi_{1}}$ and $B_{H}^{\phi_{2}}$ depicted in Figure 5, but they induce to the same 2-fold hypergraph covering $\bar{H}$ over $H$, where $\phi_{1}\left(\left(v_{1}, e_{1}\right)\right)=\phi_{1}\left(\left(e_{1}, v_{3}\right)\right)=\phi_{1}\left(\left(v_{1}, e_{3}\right)\right)=\phi_{1}\left(\left(v_{3}, e_{2}\right)\right)=\phi_{1}\left(\left(e_{2}, v_{4}\right)\right)=$ (12), $\phi_{1}\left(\left(e_{1}, v_{2}\right)\right)=\phi_{1}\left(\left(v_{2}, e_{2}\right)\right)=\phi_{1}\left(\left(e_{3}, v_{4}\right)\right)=1$, and $\phi_{2}\left(\left(v_{1}, e_{1}\right)\right)=\phi_{2}\left(\left(e_{1}, v_{3}\right)\right)=$ $\phi_{2}\left(\left(v_{1}, e_{3}\right)\right)=\phi_{2}\left(\left(v_{2}, e_{2}\right)\right)=1, \phi_{2}\left(\left(v_{3}, e_{2}\right)\right)=\phi_{2}\left(\left(e_{2}, v_{4}\right)\right)=\phi_{2}\left(\left(e_{1}, v_{2}\right)\right)=\phi_{2}\left(\left(e_{3}, v_{4}\right)\right)=$ (12).

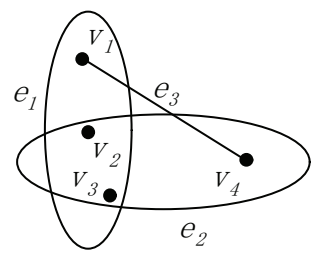

$H$

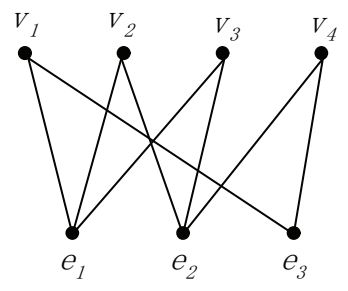

$B_{H}$

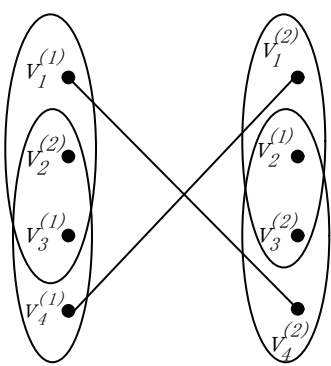

$\bar{H}$

Figure 4: The hypergraph $H$, the incidence graph $B_{H}$ of $H$, and a 2-fold hypergraph covering $\bar{H}$ over $H$

Theorem 7. Let $\bar{H}$ be any $k$-fold hypergraph covering over the hypergraph $H$. Then there is a permutation voltage assignment $\phi: E_{\overleftrightarrow{B_{H}}} \rightarrow S_{k}$ on the incidence graph $B_{H}$ of $H$ such that the derived hypergraph $H^{B_{H}^{\phi}}$ associated with $B_{H}^{\phi}$ is isomorphic to $\bar{H}$.

Proof. A hypergraph is one-to-one corresponding to its incidence graph if giving the vertices and the hyperedges with a fixed labelling. Thus, $H$ is one-to-one corresponding to its incidence graph $B_{H}$, and do $\bar{H}$ and its incidence graph $B_{\bar{H}}$. Note that $B_{\bar{H}}$ is a $k$-fold 

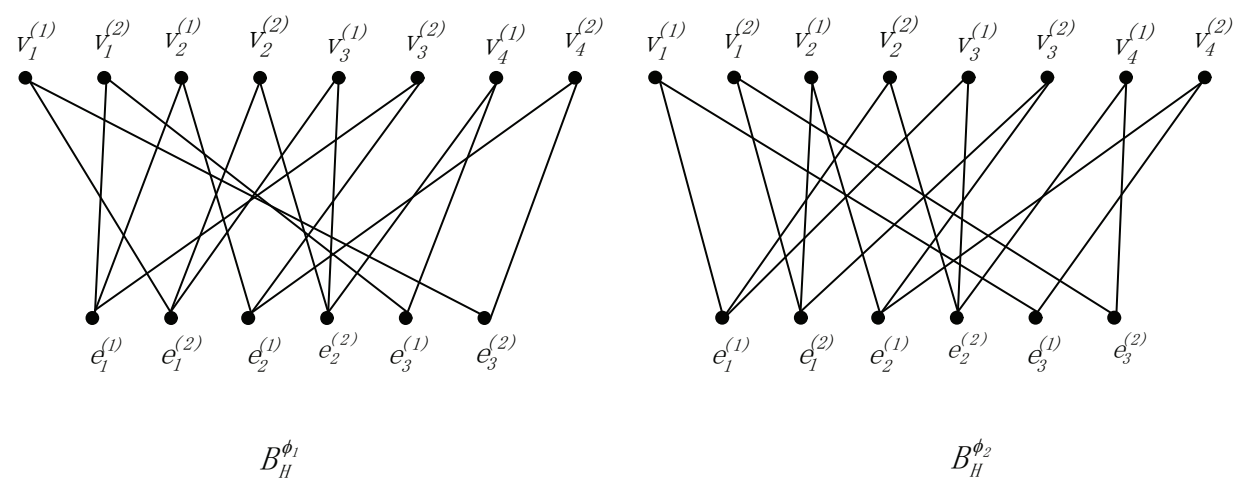

Figure 5: The derived graphs $B_{H}^{\phi_{1}}$ and $B_{H}^{\phi_{2}}$ with the permutation voltage assignments $\phi_{1}$ and $\phi_{2}$ on $B_{H}$ given in Figure 4.

covering over $B_{H}$. Then by Lemma 1 , there exists a permutation voltage assignment $\phi: E_{\overleftrightarrow{B_{H}}} \rightarrow S_{k}$ on $B_{H}$ such that $B_{H}^{\phi}$ is correspondence with $B_{\bar{H}}$. The theorem is obtained by the fact that $B_{H}^{\phi}$ being the incidence graph of $H^{B_{H}^{\phi}}$.

\section{The zeta function of a hypergraph covering}

The complex general linear group of degree $k$, denoted by $G L(k, \mathbb{C})$, is the group of all $k \times k$ invertible matrices over the complex field $\mathbb{C}$ with respect to multiplication. Let $\mathcal{H}$ be a finite group. A representation $\rho$ of the group $\mathcal{H}$ over $\mathbb{C}$ is a homomorphism from $\mathcal{H}$ to $G L(k, \mathbb{C})$, meanwhile $k$ is called the degree of the representation $\rho$. If the representation $\rho: \mathcal{H} \rightarrow G L(k, \mathbb{C})$ sends each $g \in \mathcal{H}$ to a permutation matrix, then $\rho$ is called the permutation representation of $\mathcal{H}$. The permutation representation $\mathbf{P}$ of the symmetric group $S_{k}$ sends each $g \in S_{k}$ to the $k \times k$ permutation matrix $\mathbf{P}_{g}=\left(p_{i j}^{(g)}\right)$, where

$$
p_{i j}^{(g)}= \begin{cases}1 & \text { if } i=g(j), \\ 0 & \text { otherwise. }\end{cases}
$$

The Kronecker product $A \otimes B$ of matrices $A$ and $B$ is considered as the matrix $A$ having the element $a_{i j}$ replaced by the matrix $a_{i j} B$. Let

$$
A_{1} \oplus \cdots \oplus A_{n}=\oplus_{i=1}^{n} A_{i}=\operatorname{diag}\left(A_{1}, A_{2}, \cdots, A_{n}\right)
$$

for square matrices $A_{1}, \ldots, A_{n}$, and it is written as $n \circ A$ for short when $A_{1}=\cdots=A_{n}=A$.

The following lemma is needed for the main result:

Lemma 8. [11] Let $\rho_{1}=I_{1}, \rho_{2}, \ldots, \rho_{s}$ form a complete set of inequivalent irreducible representations of $S_{k}$. Furthermore, let $f_{i}$ be the degree of $\rho_{i}$ and $m_{i}$ the multiplicity of $\rho_{i}$ in the permutation representation $\mathbf{P}$ which is defined by $E q$. (4) for each $i \in[s]$, where $f_{1}=1$. Then there exists a nonsingular matrix $M$ such that for all $g \in S_{k}$

$$
M^{-1} \mathbf{P}_{g} M=m_{1} \circ I_{1} \oplus m_{2} \circ \rho_{2}(g) \oplus \cdots \oplus m_{s} \circ \rho_{s}(g) .
$$


Theorem 9. Let $H$ be a finite hypergraph with $n$ vertices and no loops, and $G H_{c}$ be the edge-colored graph of $H$. Suppose that $H^{\widetilde{\phi}}$ is a $k$-fold hypergraph covering over $H$ associated with a strong permutation voltage assignment $\widetilde{\phi}$ on $G H_{c}$. Let $\Gamma=\left\langle\widetilde{\phi}(e) \mid e \in E_{\overleftrightarrow{G H_{c}}}\right\rangle$ be the subgroup of $S_{k}$ generated by $\left\{\widetilde{\phi}(e) \mid e \in E_{\overleftarrow{G H_{c}}}\right\}$, whose elements act as permutations on the set $[k]$. Furthermore, let $\mathbf{P}$ be a permutation representation of $\Gamma$ associated with the set $[k]$ which is defined as Eq. (4). Moreover, let $\rho_{1}=I_{1}, \rho_{2}, \cdots, \rho_{s}$ be all inequivalent irreducible representations of $\Gamma$, and the multiplicity of $\rho_{i}$ in $\mathbf{P}$ and the degree of $\rho_{i}$ be denoted by $m_{i}$ and $f_{i}$ for each $i \in[s]$, respectively. Then the zeta function of $H^{\widetilde{\phi}}$ can be decomposed as follows:

$$
\varsigma_{H^{\tilde{\phi}}}(u)^{-1}=\varsigma_{H}(u)^{-m_{1}} \prod_{i=2}^{s} \operatorname{det}\left(I_{2 l f_{i}}-u \Sigma_{g \in \Gamma}\left(\rho_{i}(g) \otimes \mathbf{A}_{g}\right)\right)^{m_{i}},
$$

where $\mathbf{A}_{g}$ is defined by Eq. (5), and $l$ is the size of $G H_{c}$.

Proof. Let $E_{\overleftarrow{G H_{c}}}=\left\{e_{1}, \ldots, e_{l}, e_{1+1}, \ldots, e_{2 l}\right\}$, where $e_{i}=e_{i-l}^{-1}$ for $i=l+1, \ldots, 2 l . G H_{c}^{\widetilde{\phi}}$ is a $k$-fold covering associated with $\widetilde{\phi}$ over $G H_{c}$. Arrange arcs of $G H_{c}^{\widetilde{\phi}}$ into $k$ blocks:

$$
e_{1}^{(1)}, \ldots, e_{l}^{(1)}, e_{1+1}^{(1)}, \ldots, e_{2 l}^{(1)} ; \ldots ; e_{1}^{(k)}, \ldots, e_{l}^{(k)}, e_{1+1}^{(k)}, \ldots, e_{2 l}^{(k)},
$$

where $e_{i}^{(j)}=\left(e_{i}, j\right)$.

Since $G H_{c}^{\widetilde{\phi}}$ is the edge-colored graph of $H^{\widetilde{\phi}}$, the vertices of the oriented line graph $H_{L}^{\widetilde{\phi}}$ of $H^{\widetilde{\phi}}$ is the arcs of $\overleftrightarrow{G H_{c}^{\phi}}$. We consider the adjacency matrix $A\left(H_{L}^{\widetilde{\phi}}\right)$ under this order. For any $g \in \Gamma$, the $2 l \times 2 l$ matrix $\mathbf{A}_{g}=\left(a_{i j}^{(g)}\right)$ is defined by

$$
a_{i j}^{(g)}= \begin{cases}1 & \text { if }\left(e_{i}, e_{j}\right) \in E_{H_{L}} \text { and } \widetilde{\phi}\left(e_{i}\right)=g \\ 0 & \text { otherwise. }\end{cases}
$$

Thus, we have

$$
A\left(H_{L}\right)=\sum_{g \in \Gamma} \mathbf{A}_{g}
$$

and from $[8]$ it follows that

$$
A\left(H_{L}^{\widetilde{\phi}}\right)=\sum_{g \in \Gamma}\left(\mathbf{P}_{g} \otimes \mathbf{A}_{g}\right)
$$

By Lemma 8, there exists a nonsingular matrix $M$ such that $M^{-1} \mathbf{P}_{g} M=m_{1} \circ \rho_{1}(g) \oplus$ $m_{2} \circ \rho_{2}(g) \oplus \cdots \oplus m_{s} \circ \rho_{s}(g)$ for all $g \in \Gamma$, where $\rho_{1}(g)=I_{1}$. Set $T=M \otimes I_{2 l}$, then by 
Eqs. (7) and (6) we have

$$
\begin{aligned}
T^{-1} A\left(H_{L}^{\widetilde{\phi}}\right) T & =\left(M \otimes I_{2 l}\right)^{-1}\left[\sum_{g \in \Gamma}\left(\mathbf{P}_{g} \otimes A_{g}\right)\right]\left(M \otimes I_{2 l}\right) \\
& =\sum_{g \in \Gamma}\left(M^{-1} \mathbf{P}_{g} M \otimes A_{g}\right) \\
& =\sum_{g \in \Gamma}\left[\left(\oplus_{i=1}^{s} m_{i} \circ \rho_{i}(g)\right) \otimes A_{g}\right] \\
& =\bigoplus_{i=1}^{s}\left\{\Sigma_{g \in \Gamma}\left[\left(m_{i} \circ \rho_{i}(g)\right) \otimes A_{g}\right]\right\} \\
& =\left[\Sigma_{g \in \Gamma}\left(I_{m_{1}} \otimes A_{g}\right)\right] \bigoplus \bigoplus_{i=2}^{s}\left\{\Sigma_{g \in \Gamma}\left[\left(m_{i} \circ \rho_{i}(g)\right) \otimes \mathbf{A}_{g}\right]\right\} \\
& =\left(m_{1} \circ A\left(H_{L}\right)\right) \bigoplus \bigoplus_{i=2}^{s}\left\{m_{i} \circ\left[\Sigma_{g \in \Gamma}\left(\rho_{i}(g) \otimes \mathbf{A}_{g}\right)\right]\right\} .
\end{aligned}
$$

Note that $\sum_{i=1}^{s} m_{i} f_{i}=k$ and $f_{1}=1$. It follows that

$$
T^{-1}\left(I_{2 l k}-u A\left(H_{L}^{\widetilde{\phi}}\right)\right) T=m_{1} \circ\left(I_{2 l}-u A\left(H_{L}\right)\right) \bigoplus \bigoplus_{i=2}^{s}\left\{m_{i} \circ\left[I_{2 l f_{i}}-u \Sigma_{g \in \Gamma}\left(\rho_{i}(g) \otimes \mathbf{A}_{g}\right)\right]\right\} .
$$

The theorem is obtained by Eq. (2).

Theorem 10. Let $H$ be a finite hypergraph with $n$ vertices and $m$ hyperedges such that each vertex is in at least two hyperedges and loops are not allowed. Furthermore, let $B_{H}$ be the incidence graph of $H$. Suppose that $\phi: E_{\overleftrightarrow{B_{H}}} \rightarrow S_{k}$ is a permutation voltage assignment on $B_{H}$ such that $\bar{H}$ is a $k$-fold hypergraph covering over $H$ corresponding to $\phi$ and $\Gamma=\left\langle\phi(e) \mid e \in E_{\overleftrightarrow{B_{H}}}\right\rangle$. Let $\mathbf{P}$ be a permutation representation of $\Gamma$ associated with the set $[k]$ which is defined as Eq. (4). Moreover, let $\rho_{1}=I_{1}, \rho_{2}, \cdots, \rho_{s}$ be all inequivalent irreducible representations of $\Gamma$, and the multiplicity of $\rho_{i}$ in $\mathbf{P}$ and the degree of $\rho_{i}$ be denoted by $m_{i}$ and $f_{i}$ for each $i \in[s]$, respectively. Then the zeta function of $\bar{H}$ can be decomposed as follows:

$$
\varsigma_{\bar{H}}(u)^{-1}=\varsigma_{H}(u)^{-m_{1}}(1-u)^{\left(k-m_{1}\right)\left(\left|E_{B_{H}}\right|-m-n\right)} \prod_{i=2}^{s} M_{i}^{m_{i}} .
$$

where $M_{i}=\operatorname{det}\left(I_{f_{i}(m+n)}-\sqrt{u} \sum_{g \in \Gamma}\left(\rho_{i}(g) \otimes \mathbf{A}_{g}\right)+u f_{i} \circ Q\left(B_{H}\right)\right)$, and $\mathbf{A}_{g}$ is defined by $E q$. (9).

Proof. We utilize Eq. (3) to prove this theorem. The proof is an analogue of the method in [6] and [7]. 
Firstly, we have $(1-u)^{\left|E_{B_{\bar{H}}}\right|-\left|V_{B_{\bar{H}}}\right|}=(1-u)^{k\left(\left|E_{B_{H}}\right|-\left|V_{B_{H}}\right|\right)}=(1-u)^{k\left(\left|E_{B_{H}}\right|-m-n\right)}$ from the definition of hypergraph coverings.

Set $V_{H}=\left\{v_{1}, v_{2}, \ldots, v_{n}\right\}$ and $E_{H}=\left\{e_{1}, e_{2}, \ldots, e_{m}\right\}$. Arrange the vertices of $B_{H}$ into 2 blocks:

$$
v_{1}, v_{2}, \ldots, v_{n} ; e_{1}, e_{2}, \ldots, e_{m},
$$

and we arrange the vertices of the incidence graph $B_{\bar{H}}$ into $2 k$ blocks:

$$
v_{1}^{(1)}, \ldots, v_{n}^{(1)} ; e_{1}^{(1)}, \ldots, e_{m}^{(1)} ; \ldots ; v_{1}^{(k)}, \ldots, v_{n}^{(k)} ; e_{1}^{(k)}, \ldots, e_{m}^{(k)},
$$

where $v_{i}^{(j)}=\left(v_{i}, j\right)$. Then the adjacency matrix $A\left(B_{\bar{H}}\right)$ and the degree matrix $D\left(B_{\bar{H}}\right)$ of $B_{\bar{H}}$ are considered under this order.

Let $\bar{H}$ be a $k$-fold hypergraph covering over $H$. We can find a permutation voltage assignment $\phi: E_{\overleftrightarrow{B_{H}}} \rightarrow S_{k}$ on $B_{H}$ such that $B_{\bar{H}}$ is corresponding to $B_{H}^{\phi}$ from Theorem 7 . For any $g \in \Gamma$, the $(n+m) \times(n+m)$ matrix $\mathbf{A}_{g}=\left(a_{i j}^{(g)}\right)$ is defined by

$$
a_{i j}^{(g)}= \begin{cases}1 & \text { if } e=\left(v_{i}, e_{j}\right) \text { or } e=\left(e_{i}, v_{j}\right) \in E_{\overleftrightarrow{B_{H}}} \text { and } \phi(e)=g \\ 0 & \text { otherwise. }\end{cases}
$$

It is easily checked that

$$
A\left(B_{H}\right)=\sum_{g \in \Gamma} \mathbf{A}_{g}
$$

Moreover, from the definition of graph coverings, the matrix $Q\left(B_{\bar{H}}\right)=D\left(B_{\bar{H}}\right)-I_{k(m+n)}$ can be expressed as:

$$
Q\left(B_{\bar{H}}\right)=I_{k} \otimes Q\left(B_{H}\right)
$$

As $B_{\bar{H}}$ is a $k$-fold covering over $B_{H}$ corresponding to $B_{H}^{\phi}$, Kwak and Lee [4] showed the adjacency matrix $A\left(B_{\bar{H}}\right)$ of $B_{\bar{H}}$ is

$$
A\left(B_{\bar{H}}\right)=\sum_{g \in \Gamma}\left(\mathbf{P}_{g} \otimes \mathbf{A}_{g}\right) .
$$

By Lemma 8, there exists a nonsingular matrix $M$ such that $M^{-1} \mathbf{P}_{g} M=m_{1} \circ \rho_{g} \oplus$ $m_{2} \circ \rho_{2}(g) \oplus \cdots \oplus m_{s} \circ \rho_{s}(g)$ for all $g \in \Gamma$, where $\rho_{g}=I_{1}$. Set $T=M \otimes I_{m+n}$. Similar to Eq. (8), by Eqs. (12) and (10) we have

$$
T^{-1} A\left(B_{\bar{H}}\right) T=\left(I_{m_{1}} \otimes A\left(B_{H}\right)\right) \bigoplus \bigoplus_{i=2}^{s}\left\{m_{i} \circ\left[\Sigma_{g \in \Gamma}\left(\rho_{i}(g) \otimes \mathbf{A}_{g}\right)\right]\right\} .
$$

Besides, we have

$$
T^{-1} Q\left(B_{\bar{H}}\right) T=\left(M^{-1} \otimes I_{m+n}\right)\left(I_{k} \otimes Q\left(B_{H}\right)\right)\left(M \otimes I_{m+n}\right)=I_{k} \otimes Q\left(B_{H}\right) .
$$


Next, we consider $\operatorname{det}\left(I_{k(m+n)}-\sqrt{u} A\left(B_{\bar{H}}\right)+u Q\left(B_{\bar{H}}\right)\right)$. Noting that $\sum_{i=1}^{s} m_{i} f_{i}=k$ and $f_{1}=1$, it follows that

$$
\begin{aligned}
& \operatorname{det}\left(I_{k(m+n)}-\sqrt{u} A\left(B_{\bar{H}}\right)+u Q\left(B_{\bar{H}}\right)\right) \\
= & \operatorname{det}\left(T^{-1}\left(I_{k} \otimes I_{m+n}-\sqrt{u} A\left(B_{\bar{H}}\right)+u Q\left(B_{\bar{H}}\right)\right) T\right) \\
= & \operatorname{det}\left(I_{\sum_{i=1}^{s} m_{i} f_{i}} \otimes I_{m+n}-\sqrt{u} I_{m_{1}} \otimes A\left(B_{H}\right) \bigoplus \bigoplus_{i=2}^{s}\left(-\sqrt{u} m_{i}\right) \circ\left(\Sigma_{g \in \Gamma}\left(\rho_{i}(g) \otimes \mathbf{A}_{g}\right)\right)\right. \\
& \left.+u I_{\sum_{i=1}^{s} m_{i} f_{i}} \otimes Q\left(B_{H}\right)\right) \quad(\text { by Eqs. }(13) \text { and }(14)) \\
= & \operatorname{det}\left(( I _ { m _ { 1 } } \otimes I _ { m + n } - \sqrt { u } I _ { m _ { 1 } } \otimes A ( B _ { H } ) + u I _ { m _ { 1 } } \otimes Q ( B _ { H } ) ) \bigoplus \bigoplus _ { i = 2 } ^ { s } \left(I_{m_{i} f_{i}} \otimes I_{m+n}\right.\right. \\
& \left.\left.-\sqrt{u} m_{i} \circ\left(\Sigma_{g \in \Gamma}\left(\rho_{i}(g) \otimes \mathbf{A}_{g}\right)\right)+u I_{m_{i} f_{i}} \otimes Q\left(B_{H}\right)\right)\right) \\
= & \operatorname{det}\left(I_{m+n}-\sqrt{u} A\left(B_{H}\right)+u Q\left(B_{H}\right)\right)^{m_{1}} \prod_{i=2}^{s} \operatorname{det}\left(I_{f_{i}(m+n)}-\sqrt{u} \Sigma_{g \in \Gamma}\left(\rho_{i}(g) \otimes \mathbf{A}_{g}\right)\right. \\
& \left.+u I_{f_{i}} \circ Q\left(B_{H}\right)\right)^{m_{i}} \\
= & \varsigma_{H}(u)^{-m_{1}}(1-u)^{-m_{1}\left(\left|E_{B_{H}}\right|-\left|V_{B_{H}}\right|\right)} \prod_{i=2}^{s} \operatorname{det}\left(I_{f_{i}(m+n)}-\sqrt{u} \Sigma_{g \in \Gamma}\left(\rho_{i}(g) \otimes \mathbf{A}_{g}\right)\right. \\
& \left.+u f_{i} \circ Q\left(B_{H}\right)\right)^{m_{i}} \quad(\text { by Eq. }(3)) .
\end{aligned}
$$

The theorem is obtained by applying Eq. (3) again.

From the above expressions of the zeta function of a hypergraph covering, the following corollary can be obtained easily:

Corollary 11. Let $H$ be a hypergraph and $\bar{H}$ be a hypergraph covering over $H$, then the zeta function of $H$ divides the zeta function of $\bar{H}$.

\section{Example}

We demonstrate the use of Theorems 9 and 10 with an example. We consider the hypergraph given in Figure 4. The permutation representation $\mathbf{P}$ of $S_{2}$ is equivalent to $I_{1} \oplus \rho_{2}$, where $\rho_{2}(1)=1$ and $\rho_{2}((12))=-1$.

The 2-fold hypergraph $\bar{H}$ is induced by the strong permutation voltage assignment $\widetilde{\phi}$ on $G H_{c}$, where $\widetilde{\phi}\left(\left(v_{1}, v_{4}\right)\right)=\widetilde{\phi}\left(\left(v_{1}, v_{2}\right)\right)=\widetilde{\phi}\left(\left(v_{2}, v_{4}\right)\right)=(12), \widetilde{\phi}\left(\left(v_{1}, v_{3}\right)\right)=\widetilde{\phi}\left(\left(v_{3}, v_{4}\right)\right)=1$, and $\widetilde{\phi}\left(\left(v_{2}, v_{3}\right)\right)=(12)$ for two arcs $\left(v_{2}, v_{3}\right)$ with distinct color. By going through the 
oriented line graph $H_{L}$ of $H$, it follows that

$$
\mathbf{A}_{1}=\left[\begin{array}{llllllllllllll}
0 & 0 & 0 & 0 & 0 & 0 & 1 & 0 & 0 & 0 & 0 & 0 & 0 & 0 \\
0 & 0 & 0 & 0 & 0 & 0 & 0 & 0 & 0 & 0 & 0 & 0 & 0 & 0 \\
0 & 0 & 0 & 0 & 0 & 0 & 0 & 0 & 0 & 0 & 0 & 0 & 0 & 0 \\
0 & 0 & 0 & 0 & 0 & 0 & 0 & 0 & 0 & 0 & 0 & 0 & 0 & 0 \\
0 & 0 & 0 & 0 & 0 & 0 & 0 & 0 & 0 & 0 & 0 & 0 & 0 & 0 \\
1 & 0 & 0 & 0 & 0 & 0 & 0 & 0 & 0 & 1 & 0 & 0 & 0 & 0 \\
0 & 0 & 0 & 0 & 0 & 0 & 0 & 0 & 0 & 0 & 0 & 0 & 0 & 0 \\
0 & 0 & 0 & 1 & 0 & 0 & 0 & 0 & 0 & 0 & 0 & 0 & 1 & 0 \\
0 & 0 & 0 & 0 & 0 & 0 & 0 & 0 & 0 & 0 & 0 & 0 & 0 & 0 \\
0 & 0 & 0 & 0 & 0 & 0 & 0 & 0 & 0 & 0 & 0 & 0 & 0 & 0 \\
0 & 0 & 0 & 0 & 0 & 0 & 0 & 0 & 0 & 0 & 0 & 0 & 0 & 0 \\
0 & 0 & 0 & 0 & 0 & 0 & 0 & 0 & 0 & 0 & 0 & 0 & 0 & 0 \\
0 & 0 & 0 & 0 & 0 & 0 & 0 & 0 & 0 & 0 & 0 & 0 & 0 & 1 \\
0 & 0 & 0 & 0 & 0 & 0 & 0 & 0 & 0 & 0 & 0 & 0 & 0 & 0
\end{array}\right]
$$

and

$$
\mathbf{A}_{(12)}=\left[\begin{array}{llllllllllllll}
0 & 0 & 0 & 0 & 0 & 0 & 0 & 0 & 0 & 0 & 0 & 0 & 0 & 0 \\
0 & 0 & 0 & 0 & 1 & 0 & 0 & 0 & 0 & 0 & 1 & 0 & 0 & 0 \\
0 & 0 & 0 & 1 & 0 & 0 & 0 & 0 & 0 & 0 & 0 & 0 & 1 & 0 \\
0 & 0 & 1 & 0 & 0 & 0 & 0 & 0 & 1 & 0 & 0 & 0 & 0 & 0 \\
0 & 0 & 0 & 0 & 0 & 0 & 0 & 0 & 0 & 0 & 0 & 0 & 0 & 1 \\
0 & 0 & 0 & 0 & 0 & 0 & 0 & 0 & 0 & 0 & 0 & 0 & 0 & 0 \\
0 & 0 & 0 & 0 & 0 & 1 & 0 & 0 & 0 & 0 & 0 & 1 & 0 & 0 \\
0 & 0 & 0 & 0 & 0 & 0 & 0 & 0 & 0 & 0 & 0 & 0 & 0 & 0 \\
0 & 0 & 0 & 0 & 0 & 0 & 1 & 0 & 0 & 0 & 0 & 0 & 0 & 0 \\
0 & 0 & 0 & 0 & 1 & 0 & 0 & 0 & 0 & 0 & 1 & 0 & 0 & 0 \\
1 & 0 & 0 & 0 & 0 & 0 & 0 & 0 & 0 & 1 & 0 & 0 & 0 & 0 \\
0 & 0 & 1 & 0 & 0 & 0 & 0 & 0 & 1 & 0 & 0 & 0 & 0 & 0 \\
0 & 0 & 0 & 0 & 0 & 0 & 0 & 0 & 0 & 0 & 0 & 0 & 0 & 0 \\
0 & 1 & 0 & 0 & 0 & 0 & 0 & 1 & 0 & 0 & 0 & 0 & 0 & 0
\end{array}\right] .
$$

So, we have

$$
\begin{aligned}
\varsigma_{H}(u)^{-1} & =\operatorname{det}\left(I_{14}-u A\left(H_{L}\right)\right) \\
& =\operatorname{det}\left(I_{14}-u\left(\mathbf{A}_{1}-\mathbf{A}_{(12)}\right)\right) \\
& =-(u-1)^{2}(1+u)\left(1+2 u+2 u^{2}\right)\left(-1+u+2 u^{3}\right) .
\end{aligned}
$$

From Theorem 9, the zeta function of $\bar{H}$ is

$$
\begin{aligned}
\varsigma_{\bar{H}}(u)^{-1} & =\varsigma_{H}(u)^{-1} \operatorname{det}\left(I_{14}-u\left(\mathbf{A}_{1}-\mathbf{A}_{(12)}\right)\right) \\
& =(u-1)^{3}(1+u)^{3}\left(1+2 u+2 u^{2}\right)\left(1-2 u+2 u^{2}\right)\left(-1+u+2 u^{3}\right)\left(1+u+2 u^{3}\right) .
\end{aligned}
$$

We also compute the zeta function of $\bar{H}$ by using Theorem 10. The permutation voltage assignment $\phi_{1}$ on $B_{H}$ is given in Example 6. We get

$$
Q\left(B_{H}\right)=\left[\begin{array}{ccccccc}
1 & & & & & & \\
& 1 & & & & & \\
& & 1 & & & & \\
& & & 1 & & & \\
& & & & 2 & & \\
& & & & & 2 & \\
& & & & & & 1
\end{array}\right] .
$$


The matrices $\mathbf{A}_{g}\left(g \in S_{2}\right)$ are listed as follows:

$$
\mathbf{A}_{1}=\left[\begin{array}{ccccccc}
0 & 0 & 0 & 0 & 0 & 0 & 0 \\
0 & 0 & 0 & 0 & 1 & 1 & 0 \\
0 & 0 & 0 & 0 & 0 & 0 & 0 \\
0 & 0 & 0 & 0 & 0 & 0 & 1 \\
0 & 1 & 0 & 0 & 0 & 0 & 0 \\
0 & 1 & 0 & 0 & 0 & 0 & 0 \\
0 & 0 & 0 & 1 & 0 & 0 & 0
\end{array}\right] \text { and } \mathbf{A}_{(12)}=\left[\begin{array}{ccccccc}
0 & 0 & 0 & 0 & 1 & 0 & 1 \\
0 & 0 & 0 & 0 & 0 & 0 & 0 \\
0 & 0 & 0 & 0 & 1 & 1 & 0 \\
0 & 0 & 0 & 0 & 0 & 1 & 0 \\
1 & 0 & 1 & 0 & 0 & 0 & 0 \\
0 & 0 & 1 & 1 & 0 & 0 & 0 \\
1 & 0 & 0 & 0 & 0 & 0 & 0
\end{array}\right] .
$$

Note that $m_{1}=m_{2}=f_{1}=f_{2}=1$. By Theorem 10 , it is calculated that

$$
\begin{aligned}
\varsigma_{\bar{H}}(u)^{-1} & =\varsigma_{H}(u)^{-1}(1-u) \operatorname{det}\left(I_{7}-\sqrt{u}\left(\mathbf{A}_{1}-\mathbf{A}_{(12)}\right)+u Q\left(B_{H}\right)\right) \\
& =(u-1)^{3}(1+u)^{3}\left(1+2 u+2 u^{2}\right)\left(1-2 u+2 u^{2}\right)\left(-1+u+2 u^{3}\right)\left(1+u+2 u^{3}\right) .
\end{aligned}
$$

The same result is obtained by utilizing two different method.

\section{Acknowledgements}

The authors are grateful to the referee for their valuable comments and helpful suggestions, which have considerably improved the presentation of this paper. In particular, the suggestion on the simplification and improvement for the proof of Lemma 3 is gratefully acknowledged.

\section{References}

[1] H. Bass. The Ihara-Selberg zeta function of a tree lattice. Internat. J. Math., 3: 717-797, 1992.

[2] J. L. Gross and T. W. Tucker. Generating all graph covering by permutation voltage assignments. Discrete. Math., 18: 273-283, 1977.

[3] M. Kotani, and T. Sunada. Zeta functions of finite graphs. J. Math. Sci. Univ. Tokyo, 7: 7-25, 2000.

[4] J. H. Kwak, and J. Lee. Characteristic polynomials of some graph bundles II. Linear and Multilinear Algebra, 32: 61-73, 1992.

[5] J. H. Kwak, and I. Sato. Zeta functions of line, middle, total graphs of a graph and their coverings. Linear Algebra Appl., 418: 234-256, 2006.

[6] H. Mizuno, and I. Sato. Characteristic polynomials of some graph coverings. Discrete Math., 142: 295-298, 1995.

[7] H. Mizuno, and I. Sato. Zeta functions of graph coverings. J. Combin. Theory Ser. B, 80: 247-257, 2000.

[8] H. Mizuno, and I. Sato. Zeta functions of oriented line graphs of graph coverings. Discrete Math., 303: 131-141, 2005. 
[9] I. Sato. A new determinant expression of the zeta function for a hypergraph. Electronic J. Combin., 16: \#R132, 2009.

[10] I. Sato. Edge zeta functions of hypergraphs. J. Appl. Math. Comput., 40: no.1-2, 209-220, 2009.

[11] J. P. Serre. Linear Representations of Finite Group. Springer-Verlag, New York, 1977.

[12] H. M. Stark, and A. A. Terras. Zeta functions of finite graphs and coverings. Adv. Math., 121: 124-165, 1996.

[13] H. M. Stark, and A. A. Terras. Zeta functions of finite graphs and coverings, II. Adv. Math., 154: 132-195, 2000.

[14] C. K. Storm. The zeta function of a hypergraph. Electronic J. Combin., 13, \#R84 2006.

[15] A. A. Terras, and H. M. Stark. Zeta functions of finite graphs and coverings, III. Adv. Math., 208: 467-489, 2007.

[16] A. A. Terras. Zeta Funtions of Graphs: A Stroll Through the Garden. Cambridge U. Press, 2011. 